Original article

\title{
Psychometric properties of the Persian version of General Self-Efficacy Scale (GSES) among substance abusers the year 2019-2020 in Kermanshah city
}

\author{
Vahid Farnia $^{\mathrm{a}}$, Roghaye Asadi ${ }^{\mathrm{b}}$, Nasrin Abdoli $^{\mathrm{a}, *}$, Farnaz Radmehr $^{\mathrm{a}}$, Mostafa Alikhani ${ }^{\mathrm{a}}$, \\ Mehdi Khodamoradi ${ }^{a}$, Behrouz Behrouz ${ }^{a}$, Safora Salemi ${ }^{a}$ \\ ${ }^{a}$ Substance Abuse Prevention Research Center, Health Institute, Kermanshah University of Medical Sciences, Kermanshah, Iran \\ ${ }^{\mathrm{b}}$ Assessment and Measurement Allameh Tabatabai University, Tehran, Iran
}

\section{A R T I C L E I N F O}

\section{Keywords:}

Substance abuse

General Self-Efficacy Scale (GSE)

Reliability

Validity

Factor structure

Correlation

\begin{abstract}
A B S T R A C T
Objectives: Self-efficacy is regarded as a significant factor in self-concept, motivational theories, and healthrelated behaviors, and can seriously influence one's level of performance. The current article seeks to determine the psychometric properties of the Persian version of the General Self-Efficacy Scale (GSES) among substance abusers.

Methods: The research method is descriptive and adopts a validation approach. 261 individuals (47 females and 214 males) were selected among the substance abusers who referred to Kermanshah Farabi Therapeutic Educational Center in 2017 in an accessible procedure, and the subjects completed the GSE Scale and AWARE (Advanced WArning of RElapse) Questionnaire-Revised Form. Exploratory and confirmatory factor analysis, Cronbach's alpha coefficient, correlation coefficient, and divergent validity were used for data analysis.

Results: The mean and standard deviation of the GSE score of the addicts was $25.79 \pm 8.54$. The findings corroborated the single-factor structure of the GSE Scale among substance abusers. This factor explained $0.67 \%$ of the total variance of the GSE Scale. Cronbach's alpha coefficient was obtained to be 0.94 . The results showed that the correlation between the GSE Scale and AWARE Questionnaire was -0.51 , and was significant at $\mathrm{p}<0.01$.

Conclusion: Given that the results showed that the GSE Scale has sufficient validity and reliability among substance abusers, it seems that this tool can be used to screen GSE screening among addicts.
\end{abstract}

\section{Introduction}

Addiction is considered a chronic and recurrent psychological disorder that is accompanied by compulsive search and consumption in spite of its detrimental consequences. ${ }^{1-3}$ One factor that is closely associated with addiction is the concept of self-efficacy. ${ }^{4,5}$ Self-efficacy is the confidence in one's ability to cope with difficult and challenging situations. ${ }^{6-8}$ Research results indicate that addicted people suffer from significantly lower self-efficacy than their non-addicted counterparts. ${ }^{9}$

Furthermore, the research performed by Kiai and Abolghasemi ${ }^{10}$ showed that with increasing self-efficacy, substance relapse temptation diminishes, and people with high quality of life and self-efficacy become less tempted to relapse.

GSE Scale has been applied in various domains including effectiveness and treatment, ${ }^{11}$ learning and academic achievement, ${ }^{12}$ physical and mental health, ${ }^{13,14}$ emotional disorders, ${ }^{14,15}$ quality of life ${ }^{16}$ and many other areas, and translated into various languages. ${ }^{16}$
One recognized questionnaire in this field is the GSE Scale designed by Schwarzer and Jerusalem in Germany and has been translated into 28 languages. ${ }^{17}$ Experimental studies using this questionnaire have shown that GSE Scale is related to other constructs predicted through cognitive-social theory. ${ }^{18}$

Apparently, there exists no questionnaire for estimating self-efficacy among addicts in Iran. However, validating this questionnaire and recognizing its application procedure can contribute to significant practical implications for researchers and therapists in the area of self-efficacy and its association with addiction and relevant consequences. Therefore, considering the importance of self-efficacy beliefs in healthrelated behaviors and psychopathology, tool validation to measure the self-efficacy construct among addicts seems necessary.

In addition, substance addicts as a vulnerable group have long been a priority in terms of performing problem-solving and research in the area of prevention, treatment and health education, and it is vital to design a relevant questionnaire. Accordingly, the current study aims to

\footnotetext{
* Corresponding author. Substance abuse prevention research center, Farabi hospital, Kermanshah University of medical sciences, Kermanshah, Iran.

E-mail address: abdolinasrin511@yahoo.com (N. Abdoli).
} 
determine the psychometric properties of the GSE Scale among substance abusers in an Iranian sample. So, the purpose of this study was therefore to validate the Persian version of the General Self-Efficacy Scale. Specifically, it is used for self-efficacy before and after the treatment of patients. It has also been used to assess changes in the lives of drug abusers after quitting.

\section{Materials and methods}

\subsection{Study population}

The research method is cross-sectional descriptive and of a validation type. The study statistical population is comprised of all substance abusers referred to Farabi Educational Center in Kermanshah in 1977. Of the target population, 261 subjects including 47 females and 214 males were selected based on the Available sampling method. The researcher-made demographic characteristics checklist, GSE Scale, and AWARE Questionnaire were used.

\subsection{Inclusion and exclusion criteria}

Inclusion criteria included the subject's satisfaction and willingness to participate in the study, at least 12 months of addiction, age range of 16-80 years, and minimum reading literacy to respond to the GSE selfreport scale. The individuals afflicted with HIV/AIDS, addiction-related psychosis and chronic diseases such as epilepsy and stroke were excluded.

\subsection{Tools}

Demographic characteristics checklist: This checklist was used by the researcher to collect data on age and sex, education level, age of substance abuse initiation, history of drug withdrawal, history of hospitalization, and non-drug counseling.

General Self-Efficacy Scale (GSES): This questionnaire was developed by Schwarzer and Jerusalem and initially included 20 items, then reduced to 10 items. The questionnaire is scored on a 4-point Likert scale (totally incorrect $=1$ to completely correct $=4$ ). The acquired scores range is $10-40$. The designers of the questionnaire reported that its reliability using Cronbach's alpha was $0.89 .{ }^{17}$

The AWARE Questionnaire (Advance WArning of Relapse): The self-report 28-items questionnaire was developed by Miller and Harris in 2000 to assess the relapse following outpatient treatment for alcohol abuse or drug dependence. The questionnaire is scored on a 7-point Likert spectrum ranging from never (score 1) to always (score 7) with the acquired score range of 28-196. Using Cronbach's alpha coefficient, the reliability and validity of the questionnaire were calculated to be 0.90 and 0.80 , respectively. ${ }^{19,20}$ this questionnaire was used for divergent validity.

\subsection{Ethical consideration}

The present study was conducted according to the Helsinki Declaration. ${ }^{26}$ Respect for the patient and confidentiality of information and obtain informed consent. The study was approved by the ethics committee of the vice chancellery of research and technology, Kermanshah University of Medical Sciences (IR.kums.REC.1396.346) and the written informed consent was obtained from each participant.

\subsection{Data analysis}

To evaluate the reliability of the GSE Scale, the internal consistency method and Cronbach's alpha coefficient were used. Using factor analysis and Varimax rotation, divergent validity and structural validity were applied to calculate the validity. The fit goodness indices included Chi-Square $\chi 2$, degrees of freedom, Standardized Mean-Square Root, Fit
Table 1

Demographic characteristics of the sample population.

\begin{tabular}{lll}
\hline Variable & Frequency (\%) & \\
\hline \multirow{2}{*}{ Age } & $\mathbf{1 6 - 2 6}$ & $50(19.2)$ \\
& $\mathbf{2 7 - 3 7}$ & $99(37.9)$ \\
& $\mathbf{3 8 - 4 7}$ & $59(22.6)$ \\
& $\mathbf{4 8 - 8 0}$ & $53(20.3)$ \\
& Total & $261(100.0)$ \\
Sex & Woman & $47(18.0)$ \\
& Man & $214(82.0)$ \\
Education & Total & $261(100.0)$ \\
& $<$ diploma & $240(92.0)$ \\
The age of the onset of addiction & $>$ diploma & $21(8.0)$ \\
& Total & $261(100.0)$ \\
& $>\mathbf{1 5}$ & $24(9.2)$ \\
& $\mathbf{1 6 - 2 0}$ & $68(26.1)$ \\
& $\mathbf{2 1 - 3 0}$ & $123(47.1)$ \\
& $\mathbf{3 1 - 4 0}$ & $35(13.4)$ \\
& $\mathbf{4 1 - 5 1}$ & $261(100.0)$ \\
\hline
\end{tabular}

Goodness Index, Goodness index correction fit, normalized fit index, and the relative fit index. Based on common criteria, any model with a fit criterion above 0.9 is regarded as an acceptable one. ${ }^{21}$ However, a cut-off point of 0.95 was set for the RMSEA fit criteria. RMSEA values less than 0.05 indicate acceptable fitness fit of the model; RMSEA values 0.05 to 0.08 indicate a near-good fit; RMSEA values 0.08 to 0.1 indicate moderate fitness, and RMSEA values greater than 0.1 indicate poor fitness of the model. Analyzes were performed via SPSS.25 and LISREL software, 8.7.

\section{Results}

$18 \%(n=47)$ of the subjects were female and $82 \%(n=214)$ were male. The highest age range was $27-37$ years with $37.9 \%(\mathrm{n}=99)$, and the lowest age range was $16-26$ years with $19.2 \%(\mathrm{n}=50) .92 \%$ $(\mathrm{n}=240)$ had a high school diploma/diploma and $8 \%(\mathrm{n}=21)$ had university degrees, and $70.3 \%$ of the age of onset of addiction in this study was young people between the ages of 16 and 30 years (Table 1).

In this part, the reliability of the research instrument using the internal consistency method and Cronbach's alpha coefficients is investigated. Based on the results, the reliability of 10 items was 0.94 (i.e. acceptable). In addition, the correlation between each item with the total score is appropriate, and omitting each item reduces the total reliability (Loop method), indicating that all items are appropriate (Table 2).

Then, in order to validate the structural validity of the questionnaire, factor analysis, and the Varimax rotation method were used. The KMO value (Kaiser-Meyer-Olkin Measure of Sampling Adequacy) was obtained to be 0.93 , indicating the adequacy of the sample. In addition, the Bartlett's Test of Sphericity was 2040.98, which was significant at $\mathrm{p}<0.01$, indicating that the items' correlation in the study population is appropriate. The correlation between GSE Scale and AWARE questionnaire was used to investigate divergent validity.

The results showed that there exists a negative correlation $(-0.51)$ between the two questionnaires $(R=-0.512$ and $P<0.01)$, respectively, and the highest factor load was related to the items 4, 6 and 9, and the lowest factor load was related to the item 2 with a factor load of 0.71 , respectively.

Further, the results indicated that one factor was extractable and accounts for about $0.67 \%$ of the GSE variable variations (Table 3).

The screen plots were used to determine the number of factors. For this purpose, given the diagram slope, the factors identified in the diagram steep slope were considered as the main factors, and the factors parallel to the slope line axis were avoided. The screen plots contributed to the identification of the one factor as the component of the Self-efficacy questionnaire. Accordingly, the screen plot below 
Table 2

Descriptive statistics, correlation matrix, reliability, and divergent validity.

\begin{tabular}{|c|c|c|c|c|c|}
\hline Element matrix & Factor & $\alpha$ & & $\mathrm{M} \pm \mathrm{SD}$ & AWARE \\
\hline $1 \mathrm{I}$ can always manage to solve difficult problems if I try hard enough & .816 & 0.939 & Total $=0.945$ & $25.79 \pm 8.54$ & $-.512^{\mathrm{a}}$ \\
\hline 2. If someone opposes me, I can find the means and ways to get what I want. & .719 & 0.944 & & & \\
\hline 3. It is easy for me to stick to my aims and accomplish my goals. & .850 & 0.942 & & & \\
\hline 4. I am confident that I could deal efficiently with unexpected events. & .849 & 0.938 & & & \\
\hline 5. Thanks to my resourcefulness, I know how to handle unforeseen situations. & .856 & 0.938 & & & \\
\hline 6. I can solve most problems if I invest the necessary effort. & .809 & 0.937 & & & \\
\hline 7. I can remain calm when facing difficulties because I can rely on my coping abilities. & .835 & 0.940 & & & \\
\hline 8. When I am confronted with a problem, I can usually find several solutions. & .835 & 0.938 & & & \\
\hline 9. If I am in trouble, I can usually think of a solution & .852 & 0.937 & & & \\
\hline 10. I can usually handle whatever comes my way. & .831 & 0.939 & & & \\
\hline
\end{tabular}

${ }^{\text {a }}$ Correlation is significant at the 0.01 level (2-tailed). $\mathrm{N}=261$, Sig. (2-tailed) $=0.000$.

Table 3

Total variance explained.

\begin{tabular}{lllll}
\hline Components & Communalities & Total & \% of Variance & Cumulative \% \\
\hline 1 & .666 & 6.705 & 67.048 & 67.048 \\
\hline
\end{tabular}

illustrates the distinction of the one factor relative to the rest, and followed by the third factor, the remaining factors are almost in the same slope (Fig. 1).

Thus, the items 1 to 10 were loaded on factor 1 were loaded on the GSES questionnaire. This factor explained 0.58 of the total variance, and as the general model was properly fitted, all of the items remained on the assumed factor (Fig. 2).

However, since only one factor was extracted and the rotation was not significant, the element matrix table and factor loading of each item on the calculated factor were also specified. The results of the matrix indicate that the highest factor loading was related to items 4, 6 and 9, respectively, and the lowest factor loadings were related to item 2 with a factor loading of 0.71 (Table 2).

Then, confirmatory factor analysis and LISREL/8.7 software were used to confirm the structure obtained. For this purpose, the designed single-factor structure was entered into the software and the fitness results of the model were studied.

Fit indices had an acceptable value; degrees of freedom were 3.13, and chi-square was 95.94 which was significant at $\mathrm{p}<0.01$. The RMSEA index was also 0.06. NFI, CFI, IFI, RMR, GFI, and AGFI indices were $0.98,0.99,0.99,0.90$ and 0.91 , respectively, which are considered appropriate (Table 4).

\section{Discussion}

The present study aimed to evaluate the psychometric properties of the GSE Scale among men and women with substance abuse in Kermanshah. A high sense of self-efficacy contributes to greater effort, resistance, and flexibility; it also influences the amount of stress and anxiety that an addict experiences when performing an activity. In addition, according to Bandura's cognitive-social learning theory, people's judgments of their abilities to succeed in a particular task strongly affects human motivation and behavior.

People with high self-efficacy believe that they can effectively influence their life events and expect more success than those with lower self-efficacy. They are not overwhelmed by their doubts and see difficult tasks as a challenge, not a threat, and usually, seek the challenge and manage to solve it. ${ }^{17,25}$

Exploratory and confirmatory factor analysis and divergent validity were used to determine the construct validity of the GSE Scale. Prior to performing the factor analysis, Kaiser-Meyer-Olkin Measure of Sampling Adequacy KMO and Bartlett's Test of Sphericity were calculated, and the results showed that the KMO value for all subjects was 0.93 .

Bartlett's test of Sphericity was 2040.98 for all subjects and was significant at $\mathrm{p}<0.01$. Thus, in addition to the adequacy of sampling, factor analysis based on the correlation matrix studied was justified and defensible.

GSE Scale is comprised of several indices including 1-Eigenvalue indices, 2- Explanation of variance, and 3-Eigenvalues diagram. Based on the screen plot which shows the Eigenvalue diagram, a factor with Eigenvalue greater than 1 was obtained, explaining $0.67 \%$ of the total variance of the GSE Scale.

Therefore, the confirmatory factor structure corroborates the singlefactor model of GSE Scale and is in line with the results obtained from the research by Schwarzer et al., ${ }^{17}$ Nilsson et al. ${ }^{22}$ and Juarez and Contreras. $^{23}$

People's performance is strongly influenced by their self-efficacy beliefs. Self-efficacy relates to what one believes and can perform under certain conditions. Different people with the same abilities in different situations, depending on whether or not self-efficacy beliefs are high or low, may operate differently. Therefore, a capable person may not be able to utilize his or her ability appropriately due to a lack of confidence in his or her ability. ${ }^{17,24,25}$ Accordingly, self-efficacy beliefs in addicts can play an essential role in the treatment and relief of the addiction

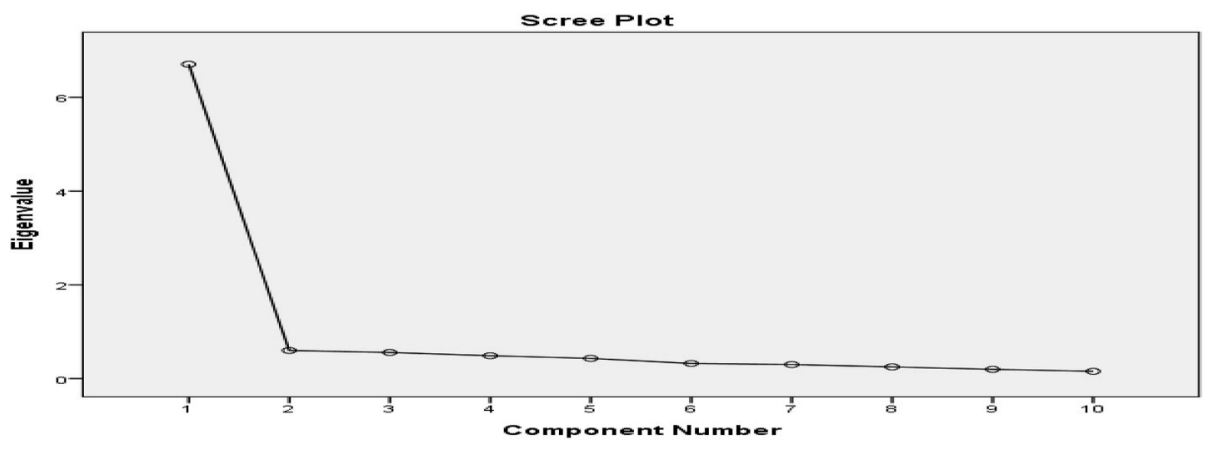

Fig. 1. Screen plot shows the one factor of the General Self-Efficacy Scale (GSES). 




Fig. 2. Standardized statistics of the GSES Score items on the single-factor model.

Table 4

Evaluation of confirmatory factor analysis indicators.

\begin{tabular}{llllllllll}
\hline Statistical title & $\mathrm{X}^{2}$ & $\mathrm{X}^{2} / \mathrm{Df}$ & $\mathrm{Df}$ & RMSEA & GFI & AGFI & CFI & NFI & SRMR \\
\hline $\begin{array}{l}\text { Desired limit } \\
\text { Estimate }\end{array}$ & & & & $0.08 \leq$ & $0.9 \geq$ & $0.9 \geq$ & $0.9 \geq$ & 0.90 \\
\hline
\end{tabular}

problem.

To calculate the divergent validity of the GSE Scale, the AWARE Questionnaire was used. The results showed that the correlation between the two questionnaires was -0.51 which was significant at $\mathrm{p}<0.01$.

However, as the GSE Score has fewer questions than the AWAER questionnaire, this makes it easier to execute, register, enter, and rate data. The reliability of the GSE questionnaire was calculated using Cronbach's alpha coefficient. The results showed that the reliability of the questionnaire was 0.94 for all subjects, indicating a satisfactory reliability.

One of the limitations of the current study is that the study was restricted to those who were referred to addiction treatment centers or hospitalized in psychiatric wards and clinics based in Farabi Educational Center in Kermanshah.

Since the data collection tool is a self-reporting tool, subjects' bias and response distortion is probable. The lack of similar studies in terms of the type of population under study made a comparison of the study slightly difficult. Therefore, it is recommended that future studies use confirmatory factor analysis more broadly in order to examine the reliability of this tool among the substance abusers population.

\section{Conclusion}

Based on the results obtained from the exploratory and confirmatory factor analysis and Cronbach's alpha, it can be concluded that the Persian version of the GSE Scale among Iranian addicts' population corroborates the single-factor characteristic of GSE Scale, and the Scale can be used as a diagnostic tool in addicts-related counseling and psychology institutions and research and therapeutic centers. So, it is used for self-efficacy before and after the treatment of patients. It has also been used to assess changes in the lives of drug abusers after quitting.

\section{Declaration of competing interest}

The authors report no conflict of interest. The authors alone are responsible for the content and writing of the paper.

\section{Acknowledgements}

The authors would like to thank the Substance abuse prevention research center and clinical Research Development Unit (CRDU) of Emam Khomeini Mohammad Kermanshahi and Farabi Hospital, Kermanshah university of Medical sciences, Kermanshah, Iran for their support, cooperation and assistance throughout the period of study.

\section{References}

1. Singer PD. Factors Associated with Long-Term Recovery from Substance Use Disorders Doctoral Dissertation Long Beach: California state university; 2016.

2. Sau M, Mukherjee A, Manna N, Sanyal S. Sociodemographic and substance use correlates of repeated relapse among patients presenting for relapse treatment at an addiction treatment center in Kolkata, India. Afr Health Sci. 2013;13(3):791-799.

3. Tam HL, Shik AWY, Lam SSL. Using expressive arts in relapse prevention of young psychotropic substance abusers in Hong Kong. Child Youth Serv Rev. 2016;60:88-100.

4. Yang C, Zhou Y, Cao Q, Xia M, An J. The relationship between self-control and selfefficacy among patients with substance use disorders: resilience and self-esteem as mediators. Front Psychiatr. 2019;10:388. https://doi.org/10.3389/fpsyt.2019.00388. eCollection.2019 Jun 12.

5. Zhang Y, Feng B, Geng W, Owens L, Xi J. "Overconfidence" versus "helplessness": a qualitative study on abstinence self-efficacy of drug users in a male compulsory drug detention center in China. Subst Abuse Treat Prev Pol. 2016;11(1):29-35.

6. Nedeljkovic M, Wepfer V, Ausfeld-Hafter B, Writz PH, Streitberger KM. Influence of general self-efficacy as a mediator in Taiji Induced stress reduction-results from a randomized controlled trial. Eur. J. Integr. Med. 2013;5(3):284-290.

7. Kim YH. Correlation of mental health problem with psychological constructs in adolescents. Int J Nurs Stud. 2003;40(2):115-124.

8. French DP. Self-efficacy and health. In: Wright JD, ed. International Encyclopedia of the Social \& Behavioral Sciences. 2 nd ed Oxford: Elsevier; 2015:509-514.

9. Silva JD, Vijayalaxmi AA. Adjustment, self-efficacy and psychosocial competency of drug-addicted adolescents. J Psychol. 2013;4(1):13-18. 
10. Kiaei T, Abolghasemi S. The relationship between quality of life, happiness, and selfefficacy with drug craving in glass consumer. Indian J. Fund. Appl. Life Sci. 2014;4(2):70-75.

11. Tang A, Tao A, Soh M, et al. The effect of interventions on balance self-efficacy in the stroke population: a systematic review and meta-analysis. Clin Rehabil. 2015;29(12):1168-1177 Dec.

12. Demirören M, Turan S, Öztuna D. Medical students' self-efficacy in problem-based learning and its relationship with self-regulated learning. Med Educ Online. 2016;21:30049https://doi.org/10.3402/meo.v21.30049.eCollection.2016 Mar 16

13. Zachariae R, O'Connor M, Lassesen B, et al. The self-efficacy in the patient-centeredness questionnaire - a new measure of medical student and physician confidence in exhibiting patient-centered behaviors. BMC Med Educ. 2015;15:150. https://doi. org/10.1186/s12909-015-0427-x Sep 15.

14. Salles A. Self-efficacy as a measure of confidence. JAMA Surg. 2017;152(5):506-507. https://doi.org/10.1001/jamasurg.2017.0035 May 1.

15. Cybulski M, Cybulski L, Krajewska-Kulak E, Cwalina U. The level of emotion control, anxiety, and self-efficacy in the elderly in Bialystok, Poland. Clin Interv Aging. 2017;12:305-314. https://doi.org/10.2147/CIA.S128717.eCollection.2017 Feb 8.

16. Mirghafourvand M, Kamalifard M, Ranjbar F, Gordani N. Relationship of breastfeeding self-efficacy with quality of life in Iranian breastfeeding mothers. J Matern Fetal Neonatal Med. 2018;31(20):2721-2728 Oct.

17. Schwarzer R, Schmitz GS, Tang C. Teacher burnout in Hong Kong and Germany: a cross-cultural validation of the maslach burnout inventory. Hist Philos Logic. 2000;13(3):309-326.
18. Vecchio GM, Gerbino M, Pastorelli C, Bove GD, Gian Vittorio Caprara GV. Multifaceted self-efficacy beliefs as predictors of life satisfaction in late adolescence. Pers Indiv Differ. 2007;43:1807-1818.

19. Kelly JF, Hoeppner BB, Urbanoski KA, Slaymaker V. Predicting relapse among young adults: psychometric validation of the advanced warning of relapse (AWARE) scale. Addict Behav. 2011;36(10):987-993. https://doi.org/10.1016/j.addbeh.2011.05. 013.

20. Miller WR, Westerberg VS, Harris RJ, Tonigan JS. What predicts relapse? Prospective testing of antecedent models. Addiction. 1996;91(Suppl):S155-S172.

21. Chen F, Curran PJ, Bollen KA, Kirby J, Paxton P. An empirical evaluation of the use of fixed cutoff points in RMSEA test statistic in structural equation models. Socio. Res. Methods. 2008;36:462-494.

22. Nilsson MH, Hagell P, Iwarsson S. Psychometric properties of the general self-efficacy scale in Parkinson's disease. Acta Neurol Scand. 2015;132(2):89-96 Aug.

23. Juarez F, Contreras F. Psychometric properties of the general self-efficacy scale in a colombian sample. Int J Psychol Res. 2008;1(2):6-12.

24. Lu FY1, Wen S2, Deng G3, Tang YL4. Self-concept mediates the relationship between childhood maltreatment and abstinence motivation as well as self-efficacy among drug addicts. May Addict Behav. 2017;68:52-58. https://doi.org/10.1016/j.addbeh. 2017.01.017 Epub 2017 Jan 6.

25. Connor JP1, Gullo MJ, Feeney GF, Kavanagh DJ, Young RM. The relationship between cannabis outcome expectancies and cannabis refusal self-efficacy in a treatment population. Jan Addiction. 2014;109(1):111-119. https://doi.org/10.1111/ add.12366 Epub 2013 Oct 28. 